\title{
Tratamento endovascular da síndrome da veia cava superior: relato de caso e revisão da literatura
}

\author{
Endovascular treatment of superior vena cava syndrome: \\ case report and review of the literature
}

\author{
M arco Aurélio Cardozo ${ }^{1}$, Eduardo Lichtenfels ${ }^{2}$, Nilon Erling Jr. ${ }^{3}$, D orvaldo P. Tarasconi ${ }^{4}$
}

\begin{abstract}
Resumo
Relatamos o caso de uma paciente portadora de síndrome da veia cava superior sintomática de origem benigna tratada pela técnica endovascular. A angiorressonância magnética pré-angioplastia evidenciou extensa trombose do tronco braquiocefálico esquerdo, da veia subclávia esquerda e obstrução da veia cava superior junto ao tronco braquiocefálico direito. A paciente realizou mastectomia radical 2 anos antes, associada à quimioterapia e radioterapia do tórax. Foram realizados angioplastia venosa e implante de stent expansível por balão. 0 resultado foi satisfatório, com alívio imediato dos sintomas devido à recanalização da veia cava superior e do tronco braquiocefálico direito. Foi instituída anticoagulação oral. A paciente permanece sem recidiva dos sintomas após 8 meses de acompanhamento. 0 tratamento endovascular é uma alternativa terapêutica com baixa morbidade e resultado satisfatório a médio prazo que pode ser oferecida aos pacientes portadores de síndrome da veia cava superior.
\end{abstract}

Palavras-chave: Angioplastia, veia cava, estenose.

\begin{abstract}
We report a case of a patient with symptomatic benign superior vena cava syndrome treated by the endovascular technique. The angiographic resonance before angioplasty showed extensive thrombosis of the left brachiocephalic trunk, left subclavian vein and superior vena cava obstruction closeto the right brachiocephal ic trunk. The patient underwent radical mastectomy 2 years ago with adjuvant chemotherapy and chest radiotherapy. Venous angioplasty and balloonexpandable stenting were performed. Satisfactory result was obtained with immediate relief of symptoms due to recanalization of the right brachiocephalic trunk and superior vena cava. O ral anticoagulation was initiated. The patient is still asymptomatic after 8 months of follow-up. The endovascular treatment is a therapeutic alternativewith low morbidity and satisfactory mid-term results that can be offered to patients with superior vena cava syndrome.
\end{abstract}

Keywords: Angioplasty, vena cava, stenosis.

marca-passos cardíacos, as fístulas arteriovenosas e os cateteres de hemodiálise ${ }^{1,2,8,9}$.

O tratamento da SV CS tem sido indicado para os pacientes sintomáticos. 0 manejo inicial constitui-se de medidas clínicas que visam ao alívio dos sintomas e a diminuição do edema no território drenado pela veia cava superior (VCS). 0 tratamento cirúrgico convencional ainda é a terapêutica mais freqüentemente utilizada para os casos refratários aos tratamentos menos invasivos, especialmenteem pacientesmaisjovens $5,9,10$.

D evido à baixa morbidade e aos resultados satisfatórios a médio prazo, as angioplastias venosas vêm se tornando o tratamento paliativo preferencial para a SV C S de origem maligna. 0 tratamento endovascular tem sido utilizado com sucesso em casos benignos de lesões pós-trombóticas, cicatrizes fibróticas pós-opera- 
tórias epós-cateteres delonga permanência, hiperplasia intimal e lesões venosas pós-radioterapia. Porém, análises de séries com maior número de pacientes e aval iação de resultados a longo prazo são necessários para estabelecer os reais benefícios dessa terapêutica aos pacientes com SV C S ${ }^{8,11-13}$.

0 objetivo deste estudo é demonstrar um caso de SV C S deorigem benignatratada com sucesso através da técnica endovascular e revisar a literatura.

\section{Relato de caso}

Paciente de 72 anos, feminina, referindo edema, rubor da face, pressão na cabeça e no pescoço e edema dos membros superiores com piora nos últimos 6 meses. Relatava exacerbação dos sintomas em posição horizontal e ao baixar a cabeça.

Foi submetida a mastectomia radical direita com esvaziamento axilar, associada à quimioterapia e radioterapia adjuvantes em razão de carcinoma de mama em 2003. A quimioterapiafoi realizada através de cateter de longa permanência instalado em veia subclávia esquerda por aproximadamente 4 meses.

Apóso diagnóstico clínico deSVCS, a paciente foi submetida a uma angiorressonância para estudo anatômico do caso e planejamento terapêutico. 0 exame demonstrou extensatrombose do tronco braquiocefálico (T BC) esquerdo, da veia subclávia esquerda eobstrução da VCS junto ao TBC direito (Figura 1).

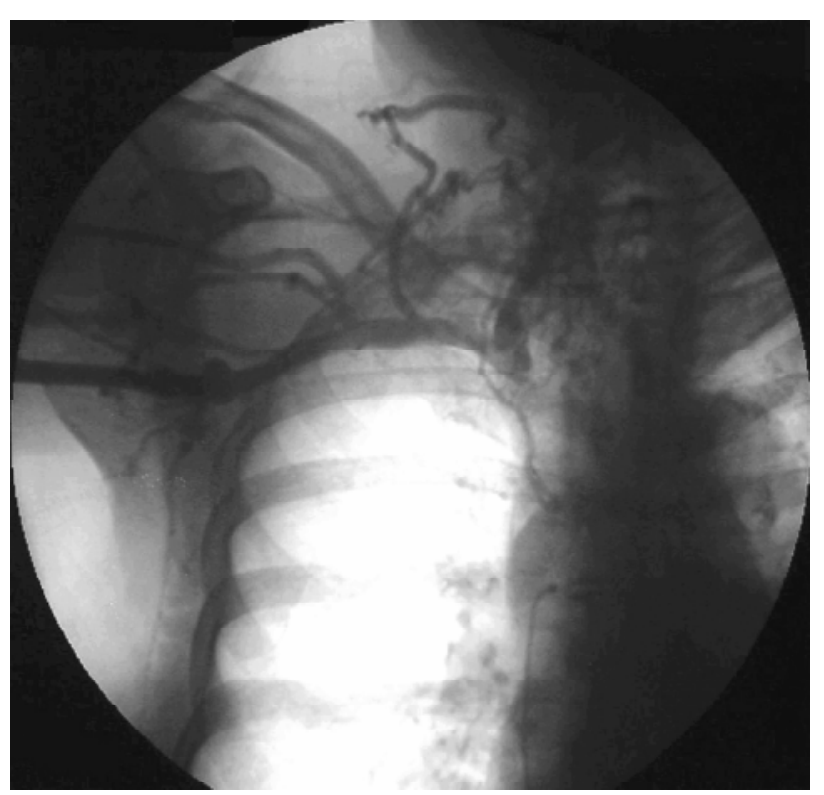

Figura 1 - Flebografia pré-operatória
0 tratamento realizado consistiu em angioplastia venosa com colocação de stent. Foi realizada a abordagem da veia braquial direita, passagem de fio guia hidrofílico pela zona de obstrução, pré-dilatação com cutting balloon de $5 \times 10 \mathrm{~mm}$ (Boston Scientific ${ }^{\circledR}$ ) e implante de stent expansível por balão de $10 \times 25 \mathrm{~mm}$ (Boston Scientific-Express LD ${ }^{\circledR}$ ) (Figura 2). Na flebografia de controle do intraoperatório, foi observada a recanalização completa do TBC direito e da VCS (Figura 3).

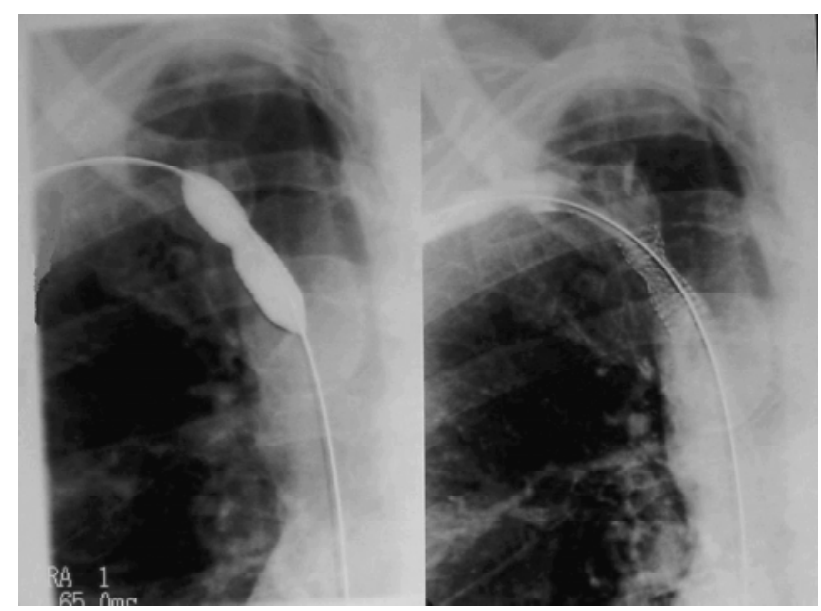

Figura 2 - Dilatação ecolocação destent balão-expansível

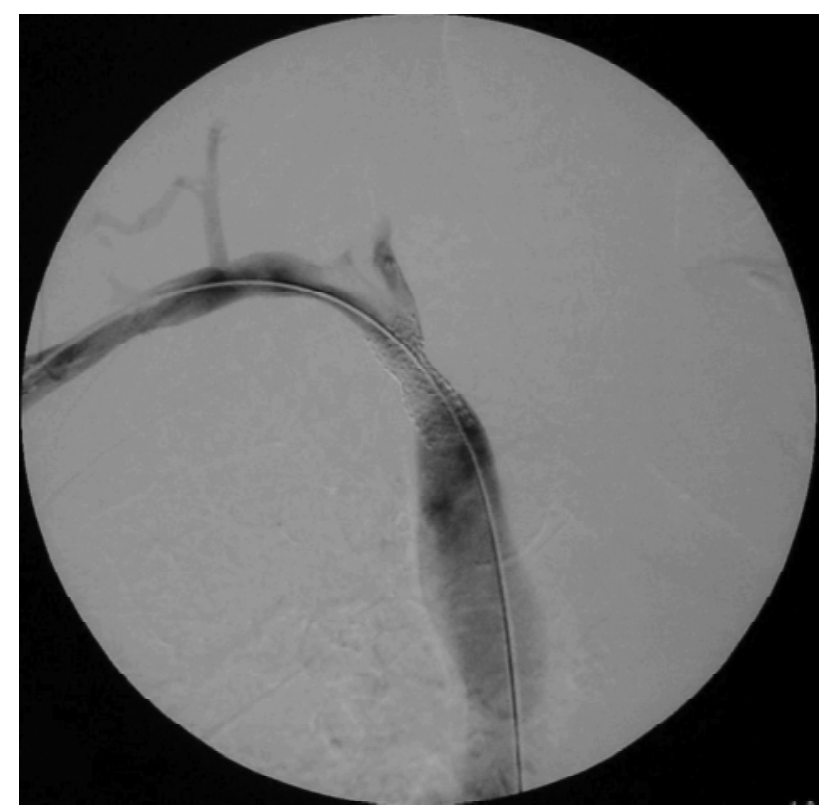

Figura 3 - Flebografia de controle 
A pacienteapresentou alívio significativo dossintomas e recebeu alta hospitalar após 48 horas, anticoagulada. Atualmente, encontra-se no oitavo mês de acompanhamento sem recidiva dos sintomas. Por solicitação dapaciente, não foi realizado examedeimagem durante 0 acompanhamento.

\section{D iscussão}

O diagnóstico etiológico é fundamental para a instituição da melhor terapêutica para o paciente com SVCS.

0 tratamento inicial da SVCS de origem benigna inclui o uso de medidas clínicas de suporte, que visam à diminuição do edema da região drenada pela VCS, reduzindo, dessa forma, os sintomas da síndrome ${ }^{5,9}$.

A indicação decirurgiaderevascularização naSV CS de origem maligna é limitada devido à alta taxa de morbimortalidade e à curta sobrevida dos pacientes 9 . $\mathrm{N}$ os casos de neoplasias mediastinais em que existe a possibilidade de cura da doença, a ressecção com revascularização venosa é a terapêutica de escolha ${ }^{14}$.

N os últimos anos, com a evolução dos cateteresbalão e dos stents, a angioplastia percutânea transluminal vem se tornado uma alternativa terapêutica importante para os pacientes portadores de SV CS. A angioplastia permite 0 alívio rápido dos sintomas sem a necessidade de procedimento cirúrgico de grande porte ${ }^{15,16}$.

A preferência entre o uso de stent auto-expansível ou expansível por balão permanece uma questão de debate. 0 s stents auto-expansíveis são mais flexíveis e calibrosos, além de se expandirem e se adaptarem à parede venosa com o passar do tempo. O sstents expansíveis por balão apresentam maior precisão durante a sualiberação emaior força radial, diminuindo o risco de recoil ${ }^{17}$. A trombólise venosa com o uso de drogas fibrinolíticas previamente à angioplastia com stent não é obrigatória, sendo uma al ternativa eficaz nos casos de oclusão extensa com presença de grande quantidade de trombos ${ }^{18}$. A pré-dilatação venosa com cateter balão da angioplastia fica reservada para as oclusões e para as estenoses muito extensas, em que pode haver a impossibilidade da passagem do sistema deliberação do stent. A colocação imediata do stent pode prevenir a embolização distal17,19.

Angioplastia com stent naSV C S deorigem maligna tem indicação nos casos com sintomas agudos ${ }^{12}$, na falha do tratamento convencional ou recidiva dos sin- tomas, o que ocorre em $50 \%$ dos $\operatorname{casos}^{3}$. Para alguns autores, essa conduta deve ser sempre considerada, por ser um tratamento paliativo para pacientes com baixa expectativa de vida ${ }^{20}$.

$\mathrm{N}$ a SV C S de origem benigna, a indicação de angioplastia ainda écontroversa ${ }^{10,11}$. 0 fato de os pacientes serem jovenseapresentarem expectativa de vida elevada gera a necessidade da realização de um procedimento com bom resultado a longo prazo. A cirurgia convencional de revascularização venosa com enxerto permanece 0 tratamento padrão com o qual todas as outras técnicas devem ser comparadas5,11,17,21. Atualmente, preconiza-se que esses pacientes devam ser submetidos inicial mente a angioplastia com ou sem a colocação de stent, associada à anticoagulação. $\mathrm{N}$ enhum desses procedimentos inviabiliza uma revascularização venosa futura10,11,15.

0 s resultados da angioplastia e colocação de stent para o tratamento da SV CS vêm sendo avaliados em diversos estudos. A completa resolução da síndrome é relatada em 68 a 100\% dos casos ${ }^{12,22-25}$. 0 sucesso clínico do tratamento varia de $55^{26}$ a $93 \% 12$. 0 sucesso técnico inicial do tratamento endovascular varia de 90 a $100 \%$ na literatura. A taxa de perviedade geral fica em torno de 77 a $85 \%$, e assistida entre 85 e 91\%, com seguimento de 17 meses ${ }^{11,18,26}$. As taxas de reestenose observadas variam de 0 a 45\%22,23. N os pacientes com lesões associadas à hemodiálise, a taxa de reestenose varia de 60 a $80 \% 27$.

Resultados a longo prazo do tratamento endovascular da SV CS foram apresentados por Smayra et al. $0 \mathrm{~s}$ autores trataram 16 pacientes com SV C S de origem maligna, cinco de origem benigna e nove causadas por hemodiálise. A perviedade em 1 ano foi de $74 \%$ para a SV C S maligna, 50\% (75\% assistida) para benigna e $22 \%$ ( $56 \%$ assistida) para os pacientes dialíticos. Complicações ocorreram em 7\% dos pacientes ${ }^{17}$.

Bornak et al. relataram uma experiência de nove pacientes tratados para SV C S de origem benigna através da técnica endovascular. A colocação de stent foi necessária em todos os casos devido ao resultado insuficiente após angioplastia isolada. A perviedade em 6 meses foi de $100 \%$, e em 12 meses foi de 67\% (100\% assistida) ${ }^{16}$.

As complicações relacionadas à colocação de stent são pouco freqüentes, variando de 7 a 19\%17,18,26. A migração do stent é uma complicação rara, mas grave, podendo levar ao óbito ${ }^{1,10,11}$. 
A anticoagulação apósa recanalização venosa não é um consenso. A maioria dosautores segue um protocolo deanticoagular os pacientes com heparina durante 0 procedimento e por 24-72 h, mantendo-os antiagregados (AAS 75-250 mg/dia) por 1-3 meses no período pós-operatório ${ }^{16}$.

0 acompanhamento deve ser realizado através do exame clínico e estudo de imagem. A ultra-sonografia com D oppler colorido possui sensibilidadee especificidade de $100 \%$ para detecção de reestenoses no segmento venoso acessível ao exame. A flebografia, a angiotomografia multi-sice e a angiorressonância magnética podem ser utilizadas para complementar a investigação eplanejar uma novaintervenção nos casos dereestenose venosa ou falência da terapêutica inicial. Embora a angiorressonância magnética apresente a desvantagem do artefato deimagem do stent metálico, elaéo método de escolha para a investigação em pacientes com perda de função renal ${ }^{16}$.

Concluímos que o tratamento endovascular é uma alternativa terapêutica que pode beneficiar pacientes com obstruções venosas centrais de origem benigna em casos sel ecionados. A intervenção endovascular é segura e apresenta bons índices de perviedade a curto e médio prazo, associada a baixas taxas de morbimortalidade.

\section{R eferências}

1. Gray $\mathrm{RJ}, \mathrm{D}$ olmatch $\mathrm{BL}, \mathrm{H}$ orton $\mathrm{KM}$, Romolo J L, ZarateAR. $M$ igration of Palmaz stents following deployment for venous stenoses related hemodialysis access. J V asc Interv Radiol. 1994;5:117-20.

2. Parish JM, M arschke RF Jr., D ines DE, Lee RE. Etiologic considerations in superior vena cava syndrome. $\mathrm{M}$ ayo $\mathrm{Clin}$ Proc. 1981;56:407-13.

3. N ieto AF, D oty D B. Superior vena cava obstruction: clinical syndrome, etiology and treatment. Curr Probl Cancer. 1986;10:441-84.

4. Perez-Soler R, M CL aughlin P, Velasquez WS, et al. Clinical features and results of management of superior vena cava syndrome secondary to lymphoma. J Clin O ncol. 1984;2: 260-6.

5. G loviczki P, V rtiska TJ. Surgical treatment of superior vena cava syndrome. In: Rutherford RB, editor. Vascular surgery. Philadelphia: WB Saunders; 2000. p. 2093-104.

6. Chen JC, Bongard F, K lein SR. A contemporary perspective on superior venacavasyndrome. AmJ Surg. 1990;160:207-11.

7. Van Putten JW, Schlosser N J, Vujaskovic Z, Leest AH, G roen HJ. Superior venacava obstruction caused by radiation induced venous fibrosis. Thorax. 2000;55:245-6.
8. Kalra M, Gloviczki P, Andrews JC, et al. O pen surgical and endovascular treatment of superior venacavasyndromecaused by nonmalignant disease. J Vasc Surg. 2003;38:215-23.

9. Cordeiro SZB, Cordeiro PB. Síndrome de veia cava superior. J Pneumol. 2002;28:288-93.

10. Cirino LMI, Coelho RF, RochalD, BatistaBPSN .T ratamento da síndrome da veia cava superior. J Bras Pneumol. 2005;31: 540-50.

11. Liddel RP, Dake MD. Endovascular treatment of chronic occlusions of large veins. In: Rutherford RB, editor. Vascular surgery. Philadelphia: W B Saunders; 2000. p. 2066-76.

12. $H$ ennequin $L M$, Fade $O$, Fays $J G$, et al. Superior vena cava stent placement: results with the $W$ allstent endoprosthesis. Radiology. 1995;196:353-61.

13. Sunder SK, EkongEA, Sivalingam K, Kumar A. Superior vena cavathrombosis dueto pacing electrodes: successful treatment with combined thrombolysis and angioplasty. Am $\mathrm{H}$ eart J. 1992;123:790-2.

14. D artevelle PG, Chapelier AR, Pastorino U, et al. Long-term follow-up after prosthetic replacement of the superior vena cava combined with resection of mediastinal-pulmonary malignant tumors. J Thorac Cardiovasc Surg. 1991;102: 259-65.

15. Yim CD, Sane SS, Bjarnason $H$. Superior vena cava stenting. Radiol Clin N orth Am. 2000;38:409-24.

16. Bornak A, Wicky S, Ris H B, Probst H, M ilesi I, Corpataux $J M$. Endovascular treatment of stenoses in the superior vena cava syndrome caused by non-tumoral lesions. Eur Radiol. 2003;13:950-6.

17. Smayra $T, O$ tal $P, C$ habbert $V$, et al. Long-term results of endovascular stent placement in the superior caval venous system. Cardiovasc Intervent Radiol. 2001;24:388-94.

18. Kee ST, Kinoshita L, Razavi MK, Nyman UR, Semba CP, $D$ ake M D. Superior vena cava syndrome: treatment with catheter-directed thrombolysis and endovascular stent placement. Radiology. 1998;206:187-93.

19. $H$ aage $P$, Vorwerk $D$, Piroth $W$, Schuermann $K$, Guenther RW. T reatment of hemodialysis-related central venousstenosis or occlusion: results of primary Wallstent placement and follow-up in 50 patients. Radiology. 1999;212:175-80.

20. Jackson JE, Brooks DM. Stenting of superior vena cava obstruction. Thorax. 1995;50:S31-6.

21. Alimi YS, Gloviczki P, Vrtiska TJ, et al. Reconstruction of superior vena cava: benefits of postoperative surveillance and secondary endovascular interventions. J Vasc Surg. 1998;27:300-1.

22. Furui S, Sawada S, Kuramoto K, et al. Gianturco stent placement in malignant caval obstruction: analysis of factors for predicting the outcome. Radiology. 1995;195:147-52.

23. O udkerk M , Kuijpers TJ, Schmitz PI, Loosveld O, deW it R. Self-expanding metal stentsfor palliativetreatment of superior vena caval syndrome. Cardiovasc Intervent Radiol. 1996;19:146-51.

24. Rosch J, Bedell JE, Putnam J, Antonovic R, Uchida B. $G$ ianturco expandablewirestents in thetreatment of superior vena cava syndrome recurring after maximum-tolerance radiation. Cancer. 1987;60:1243-6. 
25. Shah R, Sabanathan S, Lowe RA, M earns AJ. Stenting in malignant obstruction of superior vena cava. J Thorac Cardiovasc Surg. 1996;112:335-40.

26. CroweM T, D avies CH, GainesPA. Percutaneousmanagement of superior vena cava occlusions. $C$ ardiovasc I ntervent Radiol. 1995;18:367-72.

27. Zollikofer $C L$. Stent treatment in the venous circulation. In: Baert AL, Heuck FHW, Youker JE, editors. Radiology of peripheral vascular diseases. Berlin: Springer; 2000. p. 669-77.

\section{Correspondência:}

M arco Aurélio C ardozo

Rua M arquês do Pombal, 1199/401

CEP 90540-001 - Porto Alegre, RS

Tel./Fax: (51) 3337.2306

E-mail: macardozo@terra.com.br

\section{Aviso aos ex-residentes}

Se você foi residente até 2005 e ainda não está inscrito em sua Regional, procure regularizar sua situação para passar a receber o J Vasc Bras imediatamente, sem qualquer ônus.

Ajude a manter o cadastro da SBACV atualizado. 